\title{
Article
}

\section{Elovl2-ablation leads to mitochondrial membrane fatty acid remod- eling and reduced efficiency in mouse liver mitochondria}

\author{
Alexia Gómez Rodríguez ${ }^{12^{*}}$, Emanuela Talamonti ${ }^{{ }^{*}}$, Alba Naudi ${ }^{3}$, Anastasia V. Kalinovich ${ }^{1}$, Anna M. Pauter ${ }^{1}$, Gus- \\ tavo Barja ${ }^{2}$, Tore Bengtsson ${ }^{1}$, Anders Jacobsson ${ }^{1}$, Reinald Pamplona ${ }^{3}$, Irina G. Shabalina ${ }^{1}$
}

1. Department of Molecular Biosciences, The Wenner-Gren Institute, Stockholm University, SE-10691 Sweden; emanuela.talamonti@su.se, anastasia.kalinovich@su.se, annapauter@gmail.com, tore.bengtsson@su.se, anders.jacobsson@su.se

2. Department of Animal Physiology II, Faculty of Biological Sciences, Complutense University, Madrid Spain; alexiagomezrguez@gmail.com, gbarja@bio.ucm.es

3. Department of Experimental Medicine, University of Lleida - Biomedical Research Institute of Lleida (UdL-IRBLleida), E-25198 Lleida, Spain; albanaudi@gmail.com, reinald.pamplona@udl.cat

Correspondence: irina.shabalina@su.se

* these authors contributed equally to this work

\begin{abstract}
The fatty acid elongase ELOngation of Very-Long-chain fatty acids protein 2 (ELOVL2) controls the elongation of polyunsaturated fatty acids (PUFA) producing precursors for omega-3, docosahexaenoic acid (DHA), and omega-6, docosapentaenoic acid (DPAn6) in-vivo. Expectedly, Elovl2-ablation drastically reduced the DHA and DPAn6 in liver mitochondrial membranes. Unexpectedly, however, total PUFAs levels decreased further than could be explained by Elovl2 ablation. The lipid peroxidation process was not involved in PUFAs reduction since malondialdehyde-lysine (MDAL) and other oxidative stress biomarkers were not enhanced. The content of mitochondrial respiratory chain proteins remained unchanged. Still, membrane remodeling was associated with high voltage-dependent anion channel (VDAC) and adenine nucleotide translocase 2 (ANT2), a possible reflection of the increased demand on phospholipid transport to the mitochondria. Mitochondrial function was impaired despite preserved content of the respiratory chain proteins and the absence of oxidative damage. Oligomycin-insensitive oxygen consumption increased, and coefficients of respiratory control were reduced by $50 \%$. The mitochondria became very sensitive to fatty acid-induced uncoupling and permeabilization, where ANT2 is involved. Mitochondrial volume and number of peroxisomes increased as revealed by transmission electron microscopy. In conclusion, the results imply that endogenous DHA production is vital for the normal function of mouse liver mitochondria and could be relevant not only for mice but also for human metabolism.
\end{abstract}

Keywords: docosahexaenoic acid (DHA) deficiency, mitochondrial function, polyunsaturated fatty acids, membrane permeabilization, oxidative damage markers, adenine nucleotide translocase

\section{Introduction}

Dietary polyunsaturated fatty acids (PUFAs) have been shown to play important roles in human health, and mitochondria could be an essential component of PUFAs' effects [1-3]. There is growing evidence that dietary DHA profoundly affects mitochondrial membrane phospholipid composition and mitochondrial function [1]. It has been previously shown that deficiency in other PUFAs (precursors for DHA) in the diet causes uncoupling of oxidative phosphorylation in rat liver mitochondria [4]. In addition, impaired levels of dietary 18:2n-6 lead to decreased activity of the enzyme cytochrome c oxidase and reduced mitochondrial respiration in rat heart [5]. However, the role of endogenously synthesized PUFA vs. PUFA taken up from the diet is poorly investigated [6]. 
Several studies have identified the association between genetic variants in the FADS2 and ELOVL2 genes controlling endogenous PUFA synthesis and obesity-related conditions in adults and children [7-9]. However, the underlying mechanism connecting genetics and metabolism remains unknown. We have previously described the creation of ELOVL2-ablated (Elovl2 KO) mice and in vivo confirmed the fatty acid elongase ELOVL2 (ELOngation of Very Long-chain fatty acids protein 2) as a critical enzyme in the production of PUFAs with 24 carbon atoms [10]. Furthermore, Elovl2 KO mice display substantially decreased omega-3, DHA, and omega-6, DPAn6 in liver and serum [11]. Moreover, Elovl2 KO animals show metabolic changes (lower respiratory quotient) and resistance to diet-induced obesity [11], suggesting a potential role of mitochondria in regulating these metabolic processes.

Membrane phospholipids control the function of mitochondrial proteins [12-14]. The degree of unsaturation of membrane fatty acids is correlated with reactive oxygen species (ROS) production in the mitochondrial respiratory chain [15,16], leading to lipid peroxidation and subsequent damage of cellular macromolecules and their dysfunction [17]. In addition, previous studies show that changes in phospholipid composition, e.g., impaired levels of the non-bilayer-forming lipid phosphatidylethanolamine [18] or cardiolipin [4,19], affect mitochondrial function by decreasing respiratory capacity. However, little is known about how changes in the levels of specific classes of endogenous PUFAs influence mitochondrial function [6]. Also, it is essential to consider that mitochondrial fatty acid composition remodeling is partly independent of the endoplasmic reticulum where a significant amount of PUFAs are endogenously formed and Elovl2 is located [20-22]. Therefore, examining mitochondrial fatty acid composition and specifically DHA level will help unravel the origin of mitochondrial PUFA.

Thus, the present study aims to evaluate the effect of Elovl2 ablation on mitochondrial function and deduce the impaired metabolism in Elovl2 $\mathrm{KO}$ mice. We isolated liver mitochondria and analyzed their fatty acid composition, bioenergetics, and oxidative stress markers to address this question. As a result, we have found that the Elovl2 ablation in the endoplasmic reticulum drastically reduced the content of mitochondrial DHA (and other PUFAs) concomitantly with the reduction of mitochondrial efficiency despite the absence of oxidative damage and preserved content of respiratory chain proteins. The results highlight the importance of endogenous long-chain PUFAs production for proper mitochondrial function and mammalian metabolism. Furthermore, the findings presented here emphasize the importance of considering the interindividual genetic variability in endogenous long-chain PUFA production and is relevant for both dietetics and scholars working in molecular metabolism.

\section{Materials and Methods}

\section{Animals}

Twelve-week-old male Elovl2 KO mice were generated as described previously [10] and maintained on a 129SV/Sv strain backcrossed for at least ten generations. As a control, wild-type (WT) littermates of the same age were used. All animals were housed at $24{ }^{\circ} \mathrm{C}$ and maintained on a $12 \mathrm{~h}$ light:12 h dark cycle. Animals were fed ad libitum with standard chow (R70 Standard Diet, Lactamin, Stockholm, Sweden) and had free access to water. Under these standard conditions, no differences in phenotype (body weight, food intake, skin and fur appearance) were observed between WT and Elovl2 KO mice.

This study was approved by the Institutional Review Board on Animal Studies of Stockholm University and by the Animal Ethics Board of the North Stockholm region (protocol code 302/11 and date of 2014.08.25).

\section{Liver tissue collection and mitochondrial isolation}

Mice were anesthetized for 3 min with a mixture of $79 \% \mathrm{CO} 2$ and $21 \% \mathrm{O} 2$ and decapitated. One Elovl2 KO and one wild-type mouse were simultaneously processed each 
day. Livers were isolated immediately after decapitation, washed in ice-cold isolation buffer (210 mM mannitol, $70 \mathrm{mM}$ sucrose, 20 mM TES, 1 mM EDTA, pH 7.35) to remove traces of blood, cut into small pieces, and homogenized with a Potter homogenizer with a Teflon pestle in $35 \mathrm{ml}$ of the isolation buffer. Throughout the isolation process, tissues were kept at $0-2{ }^{\circ} \mathrm{C}$. Mitochondria were prepared by differential centrifugation as described previously [23]. Liver homogenates were centrifuged at $8500 \mathrm{~g}$ for $10 \mathrm{~min}$ at $2{ }^{\circ} \mathrm{C}$ using a Beckman J2-21 M centrifuge. The resulting supernatant containing floating fat, peroxisomes and other small cellular organells was discarded. The pellet was resuspended in $35 \mathrm{ml}$ of ice-cold isolation buffer with modifications (added $0.2 \%(\mathrm{w} / \mathrm{v})$ fattyacid-free BSA) to remove fat contamination and centrifuged again at $800 \mathrm{~g}$ for $10 \mathrm{~min}$ to pellet cell debris and nuclei. The resulting supernatant was centrifuged at $8500 \mathrm{~g}$ for 10 min. The final mitochondrial pellets were resuspended by homogenization in a small glass homogenizer in the same medium containing $0.2 \%$ BSA. The concentration of mitochondrial protein was measured using fluorescamine [24] with BSA as a standard.

Freshly isolated liver mitochondrial suspension was divided into three aliquots: 10 $\mu \mathrm{l}$ of mitochondrial suspension was supplemented with a protease inhibitor cocktail (CompleteTM Mini; Roche) (10:1) in an Eppendorf tube, placed in liquid nitrogen, and then stored at $-80{ }^{\circ} \mathrm{C}$ until immunoblot analysis. Another $50 \mu \mathrm{l}$ mitochondrial suspension was flushed with nitrogen gas to substitute the air in the Eppendorf tube to prevent oxidation and was used for chromatography. The majority of the freshly isolated mitochondria was kept in glass deeply immersed in ice and used for oxygen consumption measurement.

\section{Mitochondrial fatty acid composition analysis}

Fatty acids from mitochondrial membranes were analyzed as methyl ester derivatives by gas chromatography (GC) as previously described [25]. Separation was performed by a DBWAX capillary column (30 m x $0.25 \mathrm{~mm} \times 0.20 \mu \mathrm{m})$ in a GC System 7890A with a Series Injector 7683B and an FID detector (Agilent Technologies, Barcelona, Spain). Fatty acid methyl esters were identified by comparison with authentic standards (Larodan Fine Chemicals, Malmö, Sweden). Results are expressed as mol \%. The following fatty acyl indices were also calculated: saturated fatty acids (SFA); unsaturated fatty acids (UFA); monounsaturated fatty acids (MUFA); polyunsaturated fatty acids (PUFA) from n-3 and n-6 series (PUFAn-3 and PUFAn-6); and average chain length (ACL) = $[(\Sigma \%$ Total14 $\times 14)+(\Sigma \%$ Total16 $\times 16)+(\Sigma \%$ Total18 $\times 18)+(\Sigma \%$ Total $20 \times 20)+(\Sigma \%$ Total22 $\times 22)+\left(\sum \%\right.$ Total $\left.\left.24 \times 24\right)\right] / 100$. Finally, the density of double bonds in the membrane was calculated by the Double Bond Index, DBI $=[(1 \times \Sigma \mathrm{mol} \%$ monoenoic $)+(2 \times \Sigma \mathrm{mol} \%$ dienoic $)+(3 \times \Sigma \mathrm{mol} \%$ trienoic $)+(4 \times \Sigma \mathrm{mol} \%$ tetraenoic $)+(5 \times \Sigma \mathrm{mol} \%$ pentaenoic $)+$ $(6 \times \Sigma \mathrm{mol} \%$ hexaenoic $)]$.

\section{Oxidation-derived protein damage markers}

Glutamic SemiAldehyde (GSA), AminoAdipic SemiAldehyde (AASA), CarboxyEthyl-Lysine (CEL), CarboxyMethyl-Lysine (CML), and MalonDiAldehyde-Lysine (MDAL) were determined as trifluoroacetic acid methyl ester (TFAME) derivatives in acid-hydrolyzed, delipidated, and reduced mitochondrial protein samples by GC/MS [17] using an HP6890 Series II gas chromatograph (Agilent, Barcelona, Spain) with an MSD5973A Series detector and a 7683 Series automatic injector, an HP-5MS column (30$\mathrm{m} \times 0.25-\mathrm{mm} \times 0.25-\mu \mathrm{m})$, and the described temperature program [17]. Quantification was performed by internal and external standardization using standard curves constructed from mixtures of deuterated and non-deuterated standards. Analyses were carried out by selected ion-monitoring GC/MS (SIM-GC/MS). The ions used were: lysine and [2H8]lysine, $m / z 180$ and 187, respectively; 5-hydroxy-2-aminovaleric acid and [2H5]5-hydroxy-2-aminovaleric acid (stable derivatives of GSA), $m / z 280$ and 285, respectively; 6-hydroxy-2-aminocaproic acid and [2H4]6-hydroxy-2-aminocaproic acid (stable derivatives of AASA), $m / z 294$ and 298, respectively; CML and [2H4]CML, $m / z 392$ and 396, respectively; CEL and [2H4]CEL, $\mathrm{m} / \mathrm{z} 379$ and 383, respectively; and MDAL and 
[2H8]MDAL, $m / z 474$ and 482, respectively. The amounts of product were expressed as $\mu$ moles of GSA, AASA, CML, CEL, or MDAL per mol of lysine.

\section{Mitochondrial oxygen consumption}

Oxygen consumption rates were monitored with a Clark-type oxygen electrode (Yellow Springs Instrument) in a sealed chamber at $37^{\circ} \mathrm{C}$, as described previously [23] or using a high-resolution oxygraph (Oroboros Oxygraph-2K, Austria) as described previously [26]. Mitochondria were added in a concentration of $0.5 \mathrm{mg}$, to $2.0 \mathrm{ml}$ of a continuously stirred incubation medium (100 mM sucrose, $20 \mathrm{mM} \mathrm{K}$-Tes, $50 \mathrm{mM} \mathrm{KCl}, 4 \mathrm{mM}$ $\mathrm{KH}_{2} \mathrm{PO}_{4}, 2 \mathrm{mM} \mathrm{MgCl}$, $1 \mathrm{mM}$ EDTA and $0.1 \%$ fatty-acid free BSA). Basal respiration of mitochondria was measured in the presence of $5 \mathrm{mM}$ glutamate plus $3 \mathrm{mM}$ malate. To measure oxidative phosphorylation, we added $750 \mu \mathrm{M}$ ADP following $3 \mu \mathrm{g} / \mathrm{ml}$ oligomycin to inhibit phosphorylation and get the respiratory control ratio. Finally, $0.8 \mu \mathrm{M}$ of the chemical uncoupler FCCP (carbonyl cyanide 4-(trifluoromethoxy) phenylhydrazone) was added to check the maximal capacity of the electron transport chain. Oleate concentration-response traces were performed with the addition of oleate in indicated concentration in the presence of oligomycin. An inhibitor of adenine nucleotide translocase (ANT), carboxyatractyloside (CATR), was used to identify the involvement of the ANT in oleateinduced uncoupling.

\section{Transmission electron microscopy}

Transmission electron microscopy was used to visualize cellular ultrastructure. Liver slices were fixed in $2 \%$ glutaraldehyde and $0.5 \%$ paraformaldehyde in $0.1 \mathrm{M}$ sodium cacodylate buffer containing $0.1 \mathrm{M}$ sucrose ( $\mathrm{pH} 7.4$ ) at room temperature for $30 \mathrm{~min}$ and stored in a refrigerator. Fixed tissues slices were rinsed in $0.15 \mathrm{M}$ sodium cacodylate buffer ( $\mathrm{pH} 7.4$ ), postfixed in $2 \%$ osmium tetroxide ( $\mathrm{pH} \mathrm{7.4)}$ ) at $4{ }^{\circ} \mathrm{C}$ for 2 hours, dehydrated in ethanol and acetone before being embedded in LX-112 (Ladd Research Industries, Burlington, VT, USA). Sections were contrasted with uranyl acetate followed by lead citrate and examined in a Tecnai 12 Spirit BioTWIN transmission electron microscope (FEI Company, Eindhoven, The Netherlands) at $100 \mathrm{kV}$. Digital images were taken using a Veleta camera (Olympus Soft Imaging Solutions, GmbH, Münster, Germany). For estimation of mitochondrial volume ( $\mathrm{Vv}$ density of mitochondria), digital images at a final magnification of $8200 X$ were randomly taken of the cytoplasm of liver cells from tissue. Printed digital images were used, and the volume density (Vv) of mitochondria was calculated by point counting using a $2 \mathrm{~cm}$ (cytoplasm) and $1.5 \mathrm{~cm}$ (mitochondria) square lattice, according to [27]. The number of peroxisomes was estimated per $1 \mu \mathrm{m}^{2}$.

\section{Immunoblotting}

For immunoblot analysis, aliquots of freshly isolated mitochondrial suspensions were stored at $-80^{\circ} \mathrm{C}$ after supplementation with protease inhibitor cocktail (CompleteTM Mini; Roche). Protein concentrations of the thawed mitochondrial samples were re-quantified using the Lowry method. An equal volume of reducing sample buffer (0.5 M Tris- $\mathrm{HCl}$, pH 6.8, 10 \% (wt/vol) SDS, $2.5 \%$ (vol/vol) glycerol, $100 \mathrm{mM}$ dithiothreitol, and $0.5 \%$ (wt/vol) bromophenol blue) was added to each sample. Proteins were separated by SDS-PAGE in ordinary $12 \%$ and $10 \%$ polyacrylamide gel (12\% for Cox 4 , voltage-dependent anion channel (VDAC) and adenine nucleotide translocase (ANT2) and $10 \%$ for OXPHOS). Gels were loaded with $20 \mu \mathrm{g}$ of protein per sample. Equal protein loading was confirmed by ponceau red staining for the OXPHOS immunoblot membrane and with prohibitin protein as the loading control for the ANT2 immunoblot membrane. Proteins were transferred to polyvinylidene difluoride membranes (GE Healthcare Life Sciences) in $48 \mathrm{mM}$ Tris-HCl, $39 \mathrm{mM}$ glycine, 0.037 (wt/vol) SDS, and $15 \%$ (vol/vol) methanol using a semi-dry electrophoretic transfer cell (Bio-Rad Trans-Blot SD; Bio-Rad Laboratories) at $1.2 \mathrm{~mA} / \mathrm{cm} 2$ for $90 \mathrm{~min}$. After transfer, the membrane was blocked in $5 \%$ milk in Tris-buffered saline-Tween for one hour at room temperature and probed with the indicated antibodies overnight at $4{ }^{\circ} \mathrm{C}$. The immunoblot was visualized with 
appropriate horseradish peroxidase-conjugated secondary antibodies and enhanced chemiluminescence (ECL kit, GE Healthcare Life Sciences) in a charge-coupled device camera (Fuji Film, Tokyo, Japan). Antibodies used were as follows: a mixture of monoclonal antibodies against some of the structural components of oxidative phosphorylation (OXPHOS), including subunit NDUFB8 of complexes I, SDHB subunit of complex II and UQCRC2 subunit of complex III (Total OXPHOS Rodent Antibody cocktail, MS601; Mitosciences, OR, USA), dilution 1:10000; Cox4 subunit of complex IV antibody (Santa Cruz Laboratories, sc-376731), dilution 1:2000; ATP5A subunit of complex V antibody (Thermo Fisher, \#459240) dilution 1:2000 and VDAC antibody (Cell Signaling Technology, \#4661S), dilution 1:1000; adenine nucleotide translocase (ANT2) antibody (Cell Signaling, \#14671S), dilution 1:1000 and anti-Prohibitin antibody (abcam, ab75766), diluted 1:5000.

Quantification of Western blot was performed by using a standard control, a mix of all the samples, which was loaded at least twice on each gel and used as a reference to calculate the amount of protein.

\section{Chemicals}

The following chemicals were used for supplementation and additions: fatty acidfree bovine serum albumin (BSA), Fraction V (Cat\#10775835001, Roche Diagnostics $\mathrm{GmbH})$; malate (Cat\#M9138, Sigma); glutamate (Cat\#G1626, Sigma); ADP (Cat\#\#01905, Sigma). An inhibitor of adenine nucleotide translocase, carboxyatractyloside (CATR) (Cat\#216200, Calbiochem), was dissolved in $20 \mathrm{mM}$ Tes, pH 7.2. Oleate (sodium salt) (Cat\#O7501, Sigma) was dissolved in 50 \% ethanol; FCCP (Cat\#C2920, Sigma) and oligomycin (Cat\#O4876, Sigma) were dissolved in $95 \%$ ethanol. Used concentrations of ethanol do not affect mitochondrial function.

\section{Statistics}

Data were analyzed by Prism 4 software (GraphPad Software, San Diego, CA, USA) or KaleidaGraph version 5.086 by Synergy Software (Reading PA, USA). Differences between groups were analyzed by Student's t-test. A P value $<0.05$ was considered significant.

\section{Results}

\subsection{Enhanced mitochondrial volume and number of peroxisomes in Elovl $2 \mathrm{KO}$ mice}

Previous studies of Elovl2 KO mice have shown that these mice are metabolically impaired [11,28], suggesting the possible involvement of mitochondrial mechanisms. Elovl2 is expressed in the liver, and the primary metabolic mechanism may occur in liver mitochondria. Therefore an analysis of mitochondrial morphology and function became the goal of our study.

Transmission electron microscopy of mouse hepatocytes in the fixed liver slices showed a large number of primarily round mitochondrial cross-sections, which occupied a large area of the cytosol (Figure 1A-B). Although most morphological features of mitochondria were similar in wild-type and Elovl2 $\mathrm{KO}$ hepatocytes, mitochondrial volume was $21 \%$ higher in KO than in WT (Figure 1C). Thus, the higher mitochondrial volume could be one of the mechanisms of metabolic changes in Elovl2 $\mathrm{KO}$ mice.

The interesting finding by transmission electron microscopy was the increased number (by $44 \%$ ) of peroxisomes in KO compared to WT animals (Figure 1D), likely as a compensatory mechanism for the absence of Elovl2 by enhancing the steps of DHA and DPAn6 formation in the peroxisomes [29].

3.2. Elovl2 ablation leads to pronounced PUFAs deficiency in mitochondrial fatty acid composition 
Earlier studies on Elovl2 KO mice identified that fatty acid composition in both phospholipids and triglycerides of liver tissue was significantly modified, showing almost complete absence of DHA and DPAn6 [11]. Mitochondrial phospholipids contribute to total cellular phospholipids, and one can suggest that observed changes at the tissue level would be the same as at the mitochondrial level. It is known, however, that the origin of mitochondrial phospholipids is complex: some phospholipids are transferred as entire molecules from the endoplasmic reticulum, some are entirely built de-novo within mitochondria, and some are modified there [20-22]. Therefore, the mitochondrial fatty acid composition could be very different from the tissue's. Considering that Elovl2 activity occurs in the endoplasmic reticulum, we wonder how mitochondrial fatty acid composition is affected by Elovl2 ablation.

Analysis of fatty acids from mitochondrial membranes as methyl ester derivatives by gas chromatography showed that the levels of omega-3, 22:6n-3, DHA, and omega-6, 22:5(n-6), DPAn6 were significantly lower in Elovl2 KO mitochondrial extracts (DHA, 88 $\%$ reduction and DPAn6, $35 \%$ reduction) as compared with WT extracts (Figure $2 \mathrm{~A}$ ).

These results were expected and consistent with findings in whole tissue extracts [11], which directly reflects the absence of Elovl2 activity. Also, as expected, 22:5n-3 levels were significantly increased (doubled) in the Elovl2 KO mitochondria than in wildtype mitochondria (Fig. 2A), a clear indication of the steps in which Elovl2 and peroxisomal oxidation are working in omega 3 (n-3) fatty acid remodeling [29].

\section{A. WT Ilver}

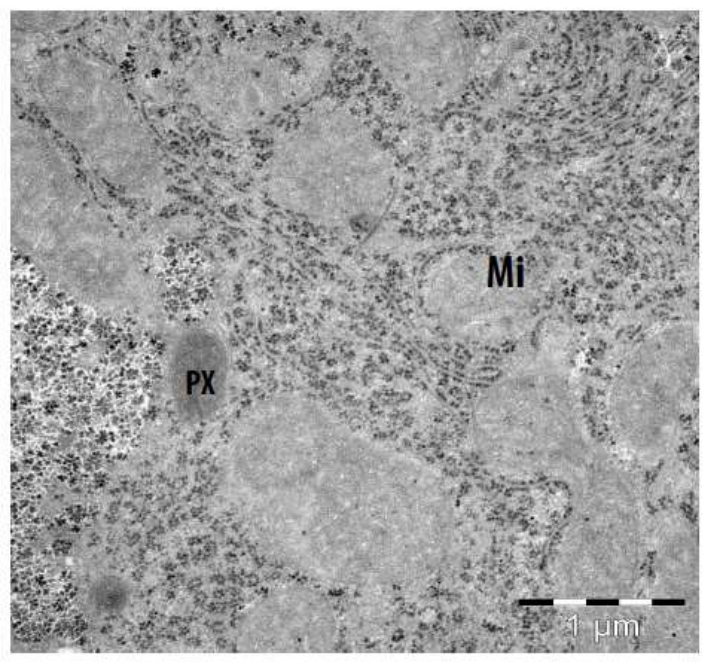

C. MItochondrlal volume

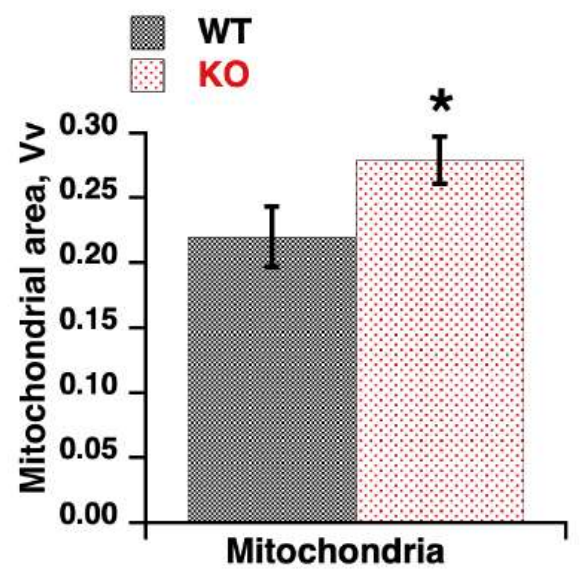

B. Elovl2 KO Ilver

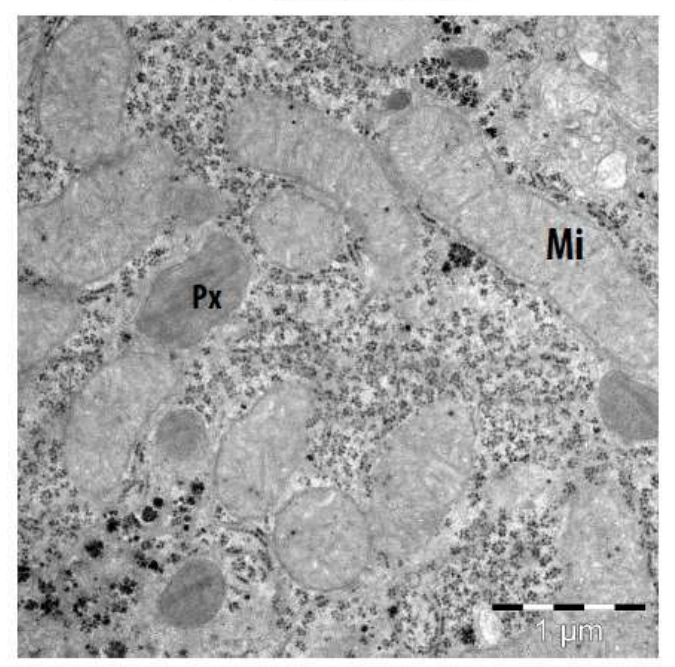

D. Number of peroxlsome

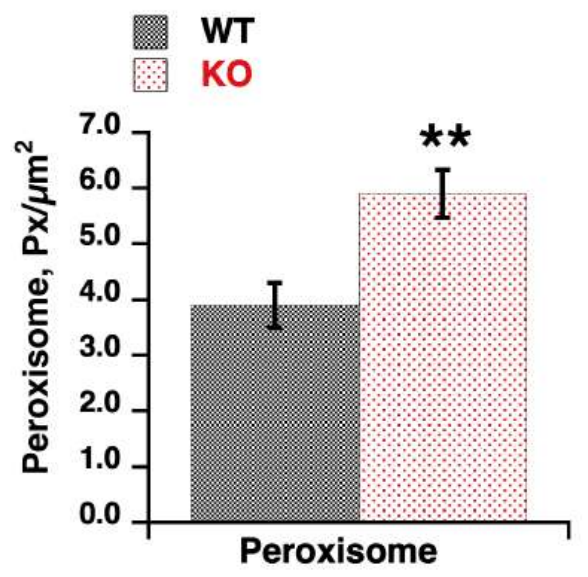


Figure 1. Morphology of liver tissue by electron microscopy.

(A, B). Transmission electron microscopy images of the liver of wild-type (WT) (A) and Elov2 KO (B) mice showing higher mitochondrial volume $(\mathrm{Mi})$ and number of peroxisomes $(P x)$ in KO compared to WT.

(C) Mitochondrial volume

(D) Peroxisome number. Values are means \pm SEM from 5 different liver samples of each genotype. Significant differences are shown between WT and Elovl2 KO mice: ${ }^{*} \mathrm{P}<0.05,{ }^{* *} \mathrm{P}<0.01$.

However, effects of Elovl2 ablation on the omega 6 (n-6) pathway were less clear. 22:4n-6 fatty acid (the closest precursor of DPAn6) levels were not increased, and levels of several other precursors of n- 6 fatty acids with various chain lengths $(18: 2 n-6 ; 20: 4 n-6)$ were even lower in Elov12 KO mitochondrial extract as compared with WT (Figure 2A). Such mitochondrial results contrast tissue results where high (not low) levels of these n-6 fatty acids have been observed, as expected [11].

A further exciting finding for mitochondria was the highest increase for fatty acid 18:1n-9 levels (38 \%), far from the Elovl2 remodeling point. These results highlight the relatively independent processes of mitochondrial fatty acid remodeling from tissue (endoplasmic reticulum) processes.

\section{A. Fatty acid profile in mitochondria}

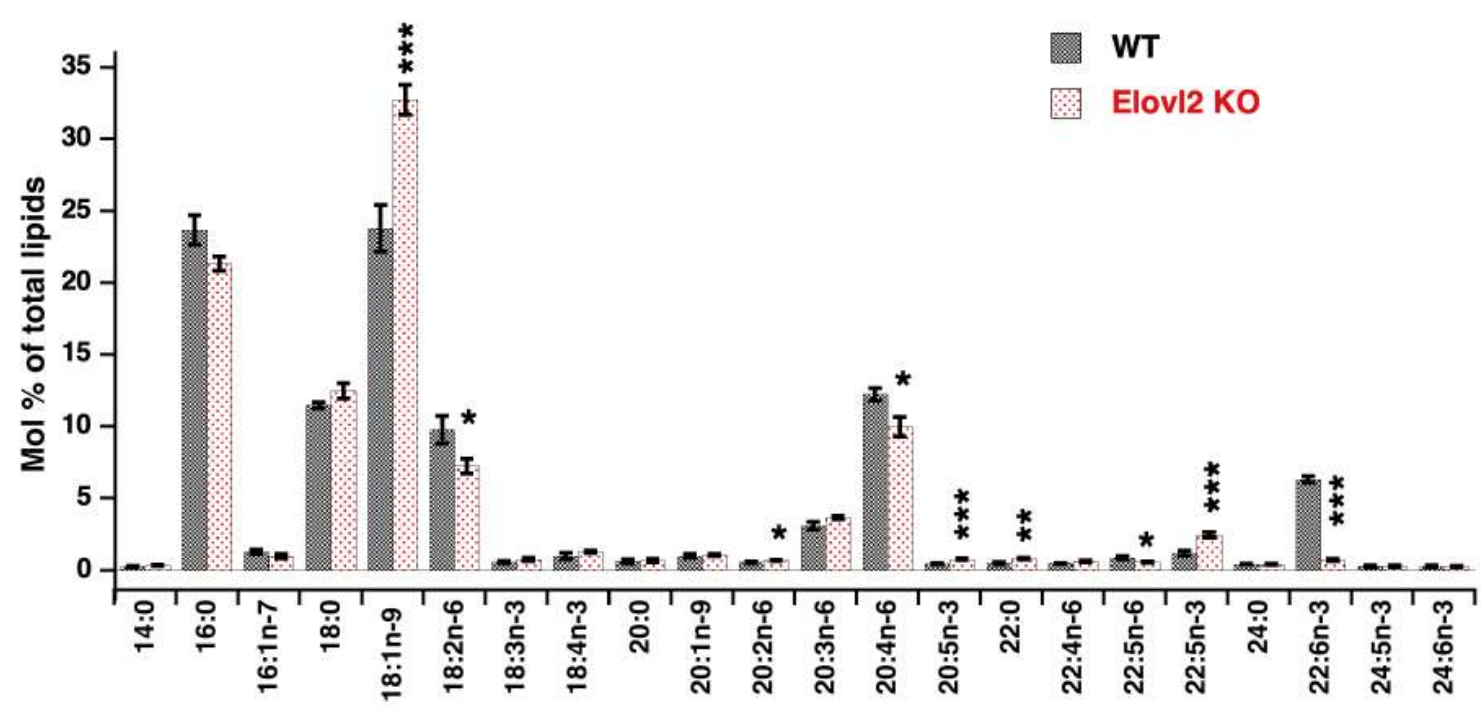

B. Fatty acid classes

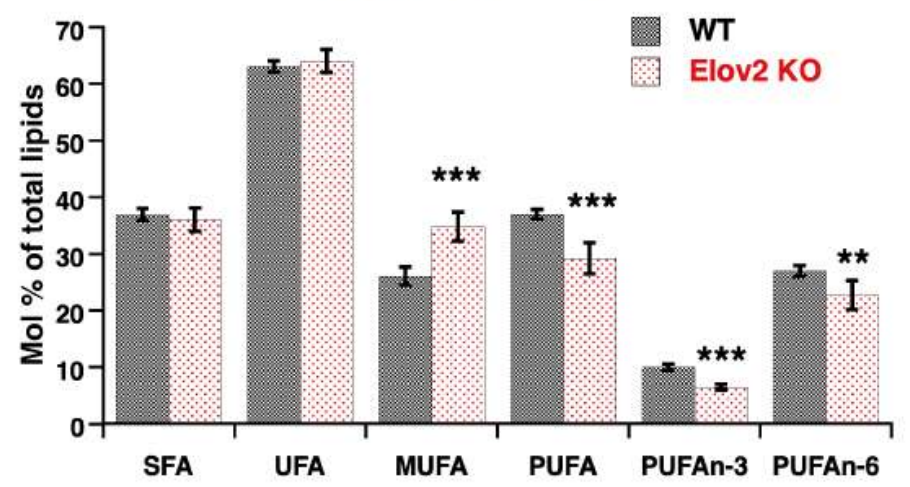

C. Double bond index

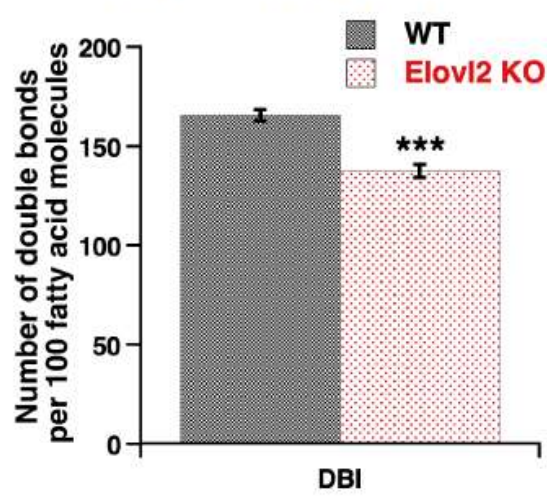

Figure 2. Fatty acid composition of liver mitochondria.

(A) Membrane fatty acid composition (mol \% of total mitochondrial lipids) in mitochondria isolated from the liver of WT and Elovl2 KO mice. 
(B) Relative amount (mol \% of total mitochondrial lipids) of different types of fatty acids: saturated fatty acids (SFA), unsaturated fatty acids (UFA), monounsaturated fatty acids (MUFA), and polyunsaturated fatty acids (PUFA).

(C) Double bond index (DBI) in membrane fatty acids. Values are means \pm SEM from 6 different mitochondrial preparations for each mouse genotype. Significant differences are shown between WT and Elovl2 KO mice: ${ }^{*} \mathrm{P}<0.05$, ${ }^{* *} \mathrm{P}<$ $0.01,{ }^{* * *} \mathrm{P}<0.001$.

As a consequence of such mitochondria-specific fatty acid remodeling, the total levels of n-3 and n-6 PUFAs decreased by $35 \%$ and $16 \%$, respectively, and the level of MUFA increased by $34 \%$ in mitochondria of Elovl2 $\mathrm{KO}$ as compared to WT (Figure 2B). In addition, the double bond index (DBI) (parameter directly reflecting fatty acid unsaturation degree) was $17 \%$ lower in the Elovl2 $\mathrm{KO}$ mice as compared to wild-type mice (Figure 2C). Furthermore, the acyl chain length (ACL) index was also significantly decreased in KO compared to WT (Supplementary Figure 1).

Thus, Elovl2 ablation significantly changed the fatty acid composition in the mitochondrial membrane, and such changes reflect both the direct effect of the absence of Elovl2 activity and an additional unknown mechanism, affecting the PUFAs double bonds.

\subsection{Absence of oxidative damage in proteins of Elovl2 $\mathrm{KO}$ mitochondria}

Fatty acid unsaturation degree is related to oxidative stress and lipid peroxidation [15]. ROS attacks the double bonds of PUFA, leading to the production of reactive aldehydes (malondialdehyde (MDA) and 4-hydroxynonenal) and reduction of double bonds in polyunsaturated fatty acids [15]. Since DBI and PUFA levels were drastically low in Elovl2 KO (Figure 2BC) and such reduction was not entirely due to the lack of Elovl 2 activity, we suggested that high lipid peroxidation activity is responsible for this reduction. It is also known that the final lipid peroxidation products, aldehydes, are highly reactive molecules and ultimately react with surrounding molecules, mainly with proteins [17]. Therefore high activity of lipid peroxidation (as a suggested mechanism of double bonds elimination) could be confirmed by the presence of high levels of oxidatively modified proteins in Elovl2 $\mathrm{KO}$ mitochondria.

To check our hypothesis, levels of oxidatively modified mitochondrial proteins were measured. However, in contrast to our prediction, the lipoxidation-dependent marker of protein modification, malondialdehyde lysine (MDAL), was significantly lower in Elovl2 $\mathrm{KO}$ mitochondria than in wild-type mitochondria; absolute values (micromoles /mole lysine) are shown in Figure 3, and the relative $28 \%$ reduction is shown in Supplementary figure 2. Thus, our hypothesis is rejected, and the mechanism behind the reduced PUFAs in Elovl2 $\mathrm{KO}$ mitochondria remains unclear.

Oxidative damage biomarkers

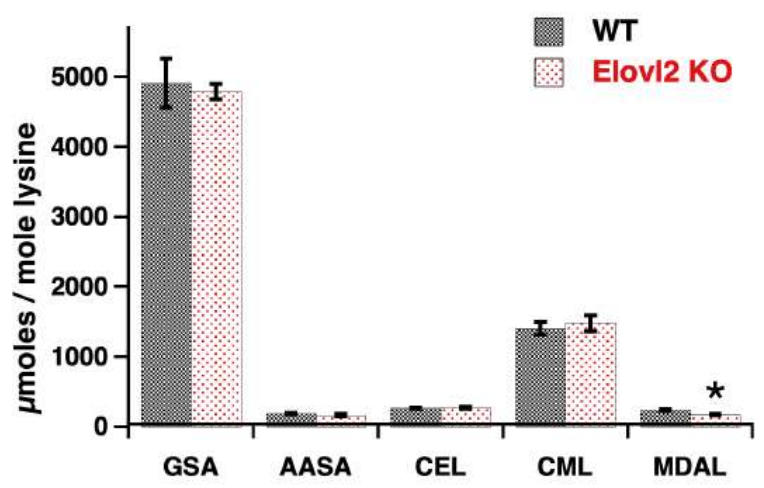

Figure 3. Oxidative damage markers.

Lipoxidation (MDAL), protein oxidation (GSA, AASA), and glycoxidation (CEL, CML) indicators in liver mitochondria from WT and Elovl2 KO mice. Values are means \pm SEM from 6 different mitochondrial preparations for each mouse genotype. Units: $\mu \mathrm{mol} / \mathrm{mol}$ lysine. Significant differences are shown between WT and Elovl2 $\mathrm{KO}$ mice, ${ }^{* *} \mathrm{P}<0.01$. 
Moreover, Elovl2 ablation entails membrane macromolecules' resistance to other types of oxidative damage. The levels of the protein markers of glycoxidation, carboxyethyl lysine (CEL) and carboxymethyl lysine (CML), as well as the specific carbonyls, glutamic and aminoadipic semialdehyde (GSA and AASA), were not significantly different between wild-type and Elovl2 KO mice (Figure 3 and Supplementary Figure 2).

Thus, due to fatty acid remodeling without ROS involvement, mitochondria in Elovl $2 \mathrm{KO}$ are PUFA deficient but protected from oxidative damage.

\subsection{Reduced mitochondrial efficiency in Elovl2 $\mathrm{KO}$ mice}

The observed differences in mitochondrial volume and morphology between Elovl2 KO and WT mice (Figure 1) as well as severe DHA deficiency (and other PUFAs decrease) in membrane composition (Figure 2) predict the possible difference in mitochondrial function. Therefore, we analyzed the content of mitochondrial functional proteins and their oxidative phosphorylation activity in intact mitochondria. 
A. Western blot

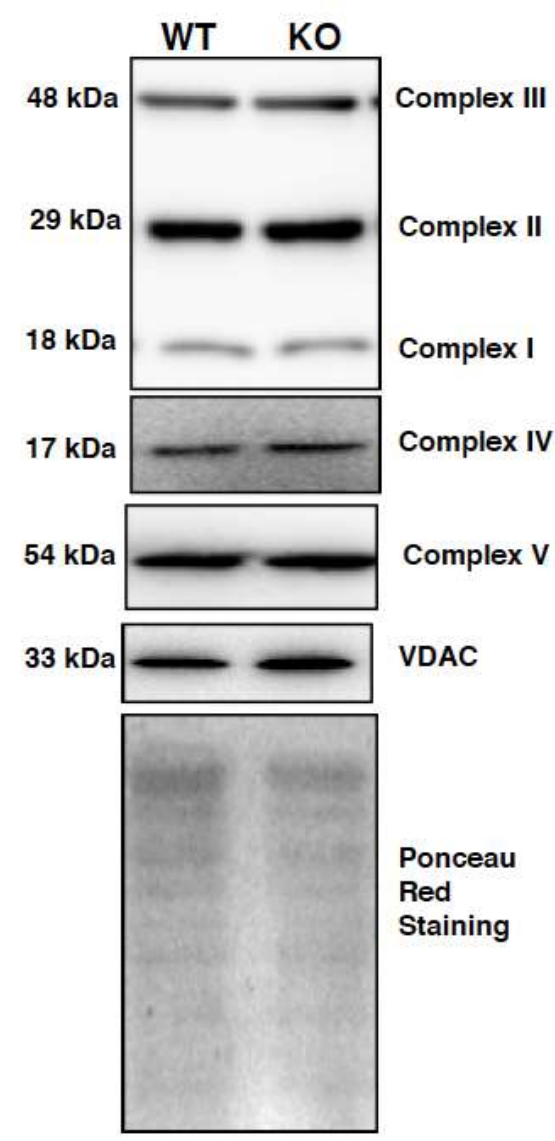

D. OXPHOS parameters

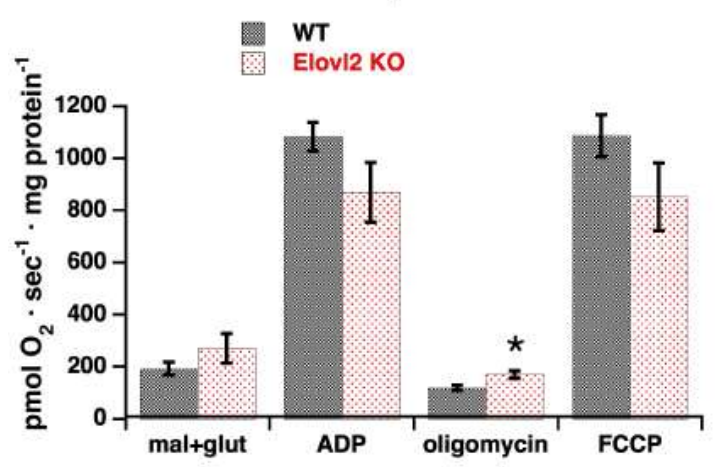

\section{B. Mltochondrlal protelns}

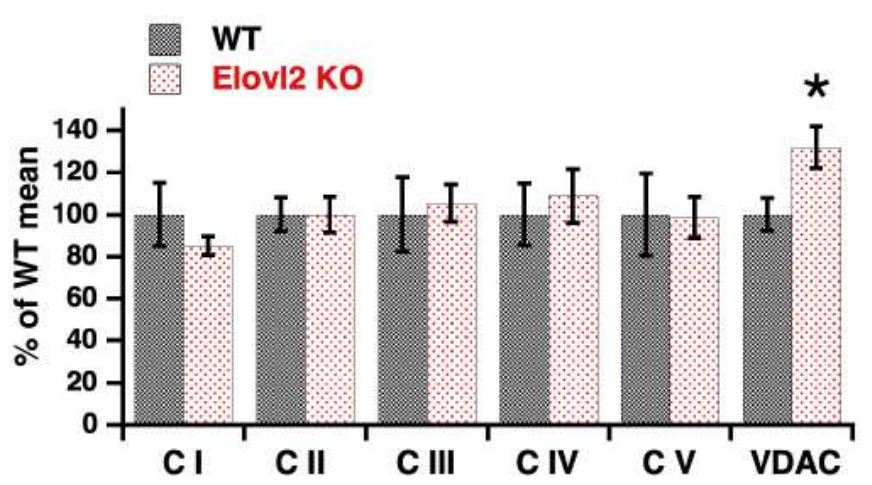

C. Mitochondrial oxygen consumption

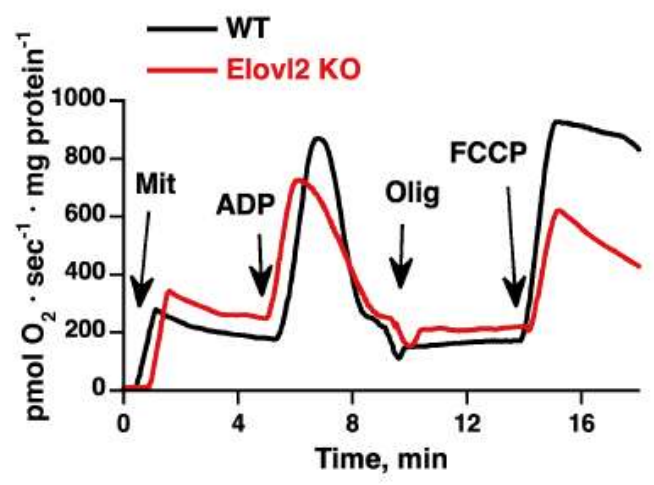

E. RespIratory Control Ratlo

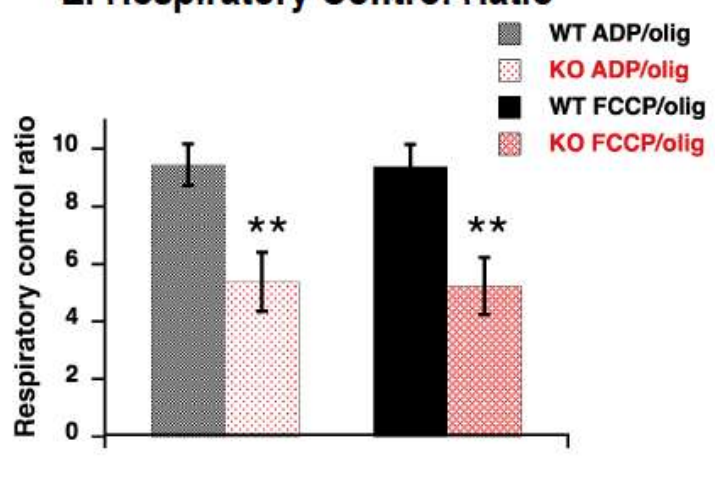

Figure 4. Mitochondrial respiratory function in WT and Elovl2 KO mice.

(A) Representative Western blots of structural components of oxidative phosphorylation and VDAC in liver mitochondria isolated from WT and Elovl2 KO mice. NDUFB8 subunit of Complex I, SDHB subunit of Complex II, UQCRC2 subunit of Complex III, COX4 subunit of Complex IV, ATP5A subunit of Complex V, and VDAC. In all cases, $20 \mu \mathrm{g}$ of mitochondrial protein were loaded per lane. Ponceau Red staining indicates equal loading of protein.

(B) Quantification of Western blot analysis. Mean of WT values was taken as $100 \%$, with all other values expressed relative to this $100 \%$. The bars are means \pm SEM from 6 independent mitochondrial preparations of each genotype.

(C) Representative oxygen consumption traces of isolated liver mitochondria from WT mice and Elovl2 KO. Additions were $0.5 \mathrm{mg}$ of liver mitochondria (Mit), $750 \mu \mathrm{M}$ ADP, $3 \mu \mathrm{g} / \mathrm{ml}$ oligomycin (Olig) and $0.8 \mu \mathrm{M}$ FCCP.

(D) Oxygen consumption rate in response to additions of malate and glutamate, ADP, oligomycin and FCCP. The values represent the means \pm SEM of 6 independent mitochondrial preparations of each group examined principally as in (A).

(E) Coefficients of respiratory control ratio, RCR (ADP/oligomycin and FCCP/oligomycin) of liver mitochondria.

In (B), (C) and (D), significant differences are shown between WT and Elovl2 KO mice, ${ }^{*} \mathrm{P}<0.05 ;{ }^{*} \mathrm{P}<0.01$. 
Western blot analysis showed levels of inner membrane respiratory chain proteins (subunits of complexes I, II, III, and IV), phosphorylation protein ATP-synthase (subunit ATP5A), and outer membrane protein VDAC (Figure 4A). The inner mitochondrial membrane protein levels were not affected by Elovl2 ablation, whereas outer membrane protein VDAC was $32 \%$ higher in liver mitochondria isolated from Elovl2 $\mathrm{KO}$ mice than from WT mice (Figure 4B). Thus, Elovl2 KO mice are one of the unique mitochondrial models where the content and intactness (absence of oxidative damage) of respiratory chain proteins were preserved, despite the changes in membrane fatty acid composition.

We, therefore, next focused on analyzing the mitochondrial functions, namely the rates of mitochondrial oxygen consumption in the presence of glutamate + malate (typical liver mitochondrial substrate linked to respiratory complex I) and under different metabolic states. After addition of substrates, ADP was added to evaluate OXPHOS capacity, followed by the ATP synthase inhibitor oligomycin to evaluate mitochondrial membrane proton leak (LEAK flux), and finally the uncoupler FCCP to measure the maximal respiratory capacity through the electron transfer system (Figure 4C). After substrate administration, the respiratory rate tended to be higher in liver mitochondria isolated from Elovl2 KO mice than from WT mice. Furthermore, respiratory rate became significantly higher in the presence of oligomycin (rate limited by the mitochondrial membrane proton leak and not by the respiratory chain) (Figure 4D). After ADP and FCCP administration, mitochondrial capacity tended to be lower in the Elovl2 $\mathrm{KO}$ mice (Figure 4D). Such changes in opposite directions indicated reduced mitochondrial efficiency and were revealed by calculating the respiratory control ratio (RCR) coefficients between ADP-stimulated and oligomycin-inhibited oxygen consumption rates and between FCCP-stimulated and oligomycin-inhibited oxygen consumption rates (Figure $4 \mathrm{E})$. Mitochondrial RCR coefficients in Elovl2 KO mice were significantly lower $(\approx 55 \%)$ than in WT mice (Figure 4E).

Given these results, we conclude that a decrease in long-chain PUFA content, especially DHA, in the mitochondrial membrane leads to decreased mitochondrial efficiency. Leaky (inefficient) mitochondria and increased total mitochondrial volume in liver tissue can be the mechanisms responsible for metabolic changes in Elovl2 $\mathrm{KO}$ mice.

\subsection{Elovl2 KO mitochondria are more sensitive to fatty acid-induced uncoupling}

Having in hand an excellent mitochondrial model with increased basal proton leak and preserved respiratory chain proteins, we took the opportunity to study the mechanism of mitochondrial uncoupling in depth. We next looked at a mitochondrial inducible proton leak by natural inducers of proton leak, fatty acids. The titration of mitochondria with oleate was performed in Elovl2 KO mitochondria in parallel with wild-type mitochondria (Figure 5A), and significant differences in concentration-response curves were observed (Figure $5 \mathrm{AB}$ ). In particular, the $\mathrm{KO}$ mitochondria were more sensitive to the uncoupling effect of oleate at concentration $60 \mu \mathrm{M}$ oleate, where significantly higher oxygen consumption was observed in $\mathrm{KO}$ mitochondria than in WT mitochondria) (Figure 5B).

Furthermore, although no difference between magnitudes of maximal rate of responses was observed in mitochondria of Elovl2 KO and WT mice (Figure 5B), the estimated EC50 (the dose induced $50 \%$ of response) was significantly higher in Elovl2 KO compared to WT (Figure 5C). Also, notably, Elovl2 KO mitochondria are more easily inhibited (permeabilized) by a high concentration of oleate: $100 \mu \mathrm{M}$ oleate induced profound inhibition of oxygen consumption (detergent-like effect) in Elovl2 KO mitochondria but not in WT mitochondria (Figure 5B).

Thus, liver mitochondria deficient in PUFAs but with preserved respiratory chain and without oxidatively damaged proteins have both high basal and inducible proton leak and easily undergo permeabilization. 


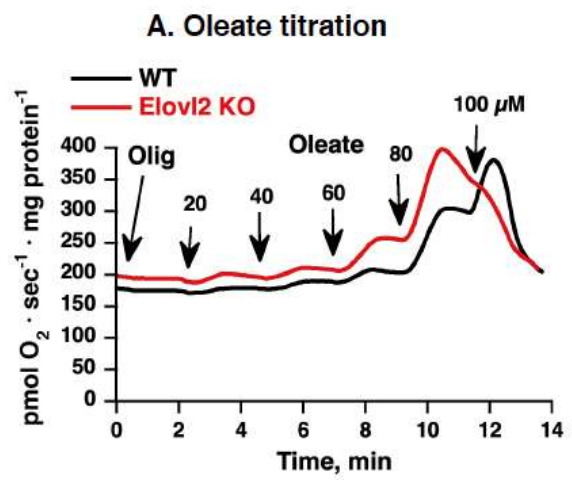

B. Dose-response curves

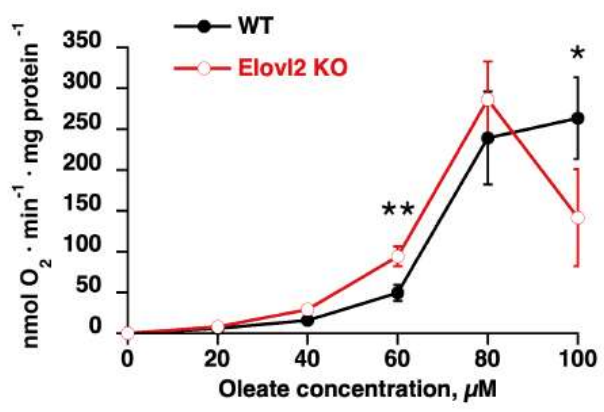

C. EC50 oleate

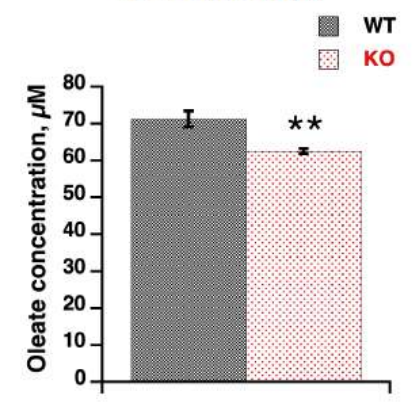

Figure 5. Fatty acid-induced uncoupling in Elovl2 KO mitochondria.

(A) Representative oxygen consumption traces showing oleate effects in liver mitochondria from wild-type (WT, black line) and Elovl2 $\mathrm{KO}$ (KO, red line). The mitochondria were incubated with BSA and malate and glutamate, then with $3 \mu \mathrm{g} / \mathrm{ml}$ oligomycin (Olig), and titrated with oleate as indicated by arrows. Each addition of oleate was $20 \mu \mathrm{M}$ (the final nominal concentrations of oleate are shown on the graph).

(B) Dose-response curves for oleate were based on experiments as those shown in (A). The values were calculated as the increase of respiratory rate above oligomycin level.

(C) Comparison between the apparent EC50 concentrations for respiratory stimulation by oleate in WT and Elovl2 KO mitochondria. Values were obtained from the dose-response curves shown in (A) by manual interpolations for the oleate concentration, which yielded $50 \%$ of the maximal respiratory value.

In (B) and (C), the values are the means \pm SEM from 4 independent liver mitochondria isolations of each genotype performed in parallel. Significant differences are shown between WT and Elovl2 KO mice: ${ }^{*} \mathrm{P}<0.05,{ }^{* *} \mathrm{P}<0.01$. 
To get further insight into the mechanism of mitochondrial inefficiency, we investigated adenine nucleotide translocase (ANT), a membrane protein shown to be involved in mitochondrial uncoupling in the liver [30-32].

A. Representatlve oxygen consumption trace

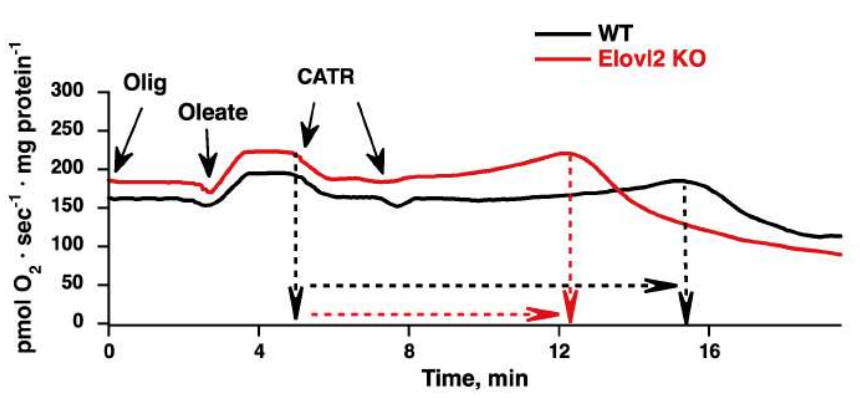

C. $\triangle$ CATR effect

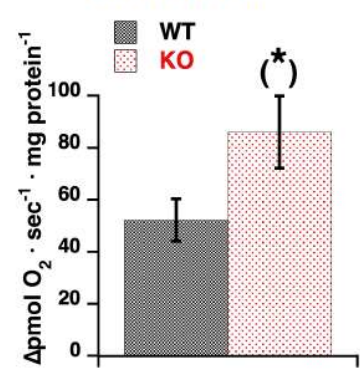

D. TIme of permeabIIIzation

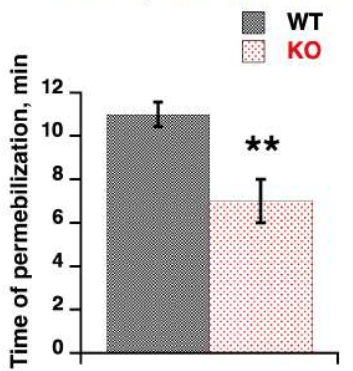

B. Effect of CATR on oleate

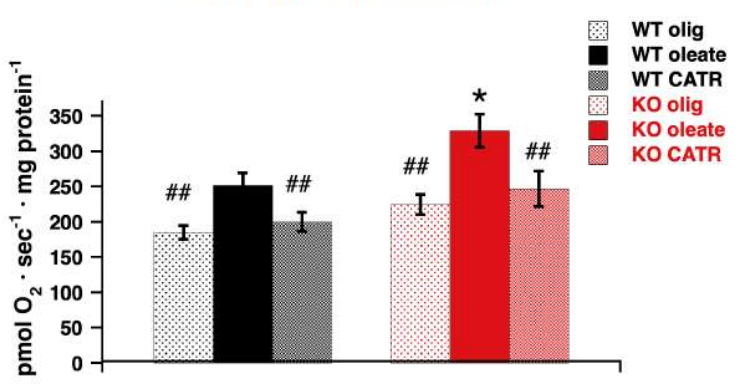

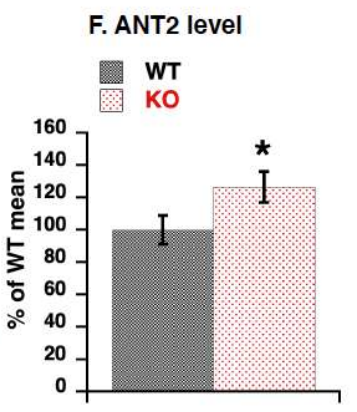

Figure 6. ANT2 protein level and CATR effect on fatty acid-induced uncoupling in wild-type and Elovl2 KO mitochondria.

(A) Representative oxygen consumption traces showing effects of $60 \mu \mathrm{M}$ oleate and $2 \mu \mathrm{M}$ CATR in liver mitochondria. The mitochondria were incubated as in Figure 5(A) with BSA and malate and glutamate, then with $3 \mu \mathrm{g} / \mathrm{ml}$ oligomycin (Olig), and oleate was added as a single $60 \mu \mathrm{M}$ dose to induce detectable respiration; then treated with $1 \mu \mathrm{M}$ carboxyatractyloside (CATR) twice as indicated by arrows. Dashed black (WT) and red (KO) arrows indicated different time durations for development of permeabilization-like phenomena.

(B) Compilation of experiments performed as in A. The bars are means \pm SEM from 4 independent mitochondrial preparations of each genotype. Significant differences are shown between WT and Elovl2 KO mice, ${ }^{*} \mathrm{P}<0.05$ and between oleate-induced respiration and other respiratory states (oligomycin-insensitive and CATR-inhibited), ${ }^{\#} \mathrm{P}<0.01$.

(C) CATR effect was estimated as $\Delta$ between rate of oleate-stimulated and CATR-inhibited rate. The bars are means \pm SEM from 4 independent mitochondrial preparations of each genotype. $\left(^{*}\right.$ ) indicates $\mathrm{P}=0.06$ (almost reaching significance between WT and Elovl2 KO mice).

(D) Time to permeabilization after CATR addition. The time period between adding the first dose of CATR and the spontaneously developed peack of oxygen consumption was measured in minutes for each mitochondrial preparation as shown in (A), and means \pm SEM from 4 independent mitochondrial preparations of each genotype are presented by bars. Significant differences are shown between $\mathrm{WT}$ and Elovl2 $\mathrm{KO}$ mice, ${ }^{* *} \mathrm{P}<0.01$.

(E) Representative Western blot of ANT2 protein from WT and Elovl2 KO liver mitochondria. Prohibitin is used as a loading control. In all cases, $20 \mu \mathrm{g}$ of mitochondrial protein were loaded per lane.

(F) Quantification of Western blot analysis. As a standard control, a mix of all the samples was loaded at least twice on each gel and used as a reference to calculate the amount of protein. These raw values were used for statistical analysis. For graphical presentation, normalization was performed: mean of WT values was taken as $100 \%$, and all other values are expressed relative to this $100 \%$. The bars are means \pm SEM from 6 independent mitochondrial preparations of each genotype. Significant differences are shown between WT and Elovl2 KO mice, ${ }^{*} \mathrm{P}<0.05$.

To examine ANT's potential involvement in the observed high fatty acid-induced uncoupling in mitochondria of Elovl2 $\mathrm{KO}$ mice, we used carboxyatractyloside (CATR), a specific and irreversible inhibitor of ANT [33,34] (Figure 6A). We found that a substantial fraction of the oleate-induced increase in oxygen consumption in liver mitochondria 
was inhibited by CATR (Figure 6AB). This inhibitory effect appeared to be higher in KO mitochondria compared to WT (Figure 6C), suggesting that ANT mediates the significant part of fatty acid-induced uncoupling effect in Elovl2 $\mathrm{KO}$ mitochondria.

While establishing the CATR effect, we noticed that prolonged exposure of mitochondria to oleate (of a notably small $60 \mu \mathrm{M}$ concentration, below the concentration of $100 \mu \mathrm{M}$ showing detergent-like effect in Figure 5A) and CATR induced a spontaneously developed change in oxygen consumption: the initial stable oxygen consumption slowly increased for 6-12 min and exhibited a short peak of oxygen consumption, followed by quickly developed inhibition (Figure 6A). Such a pattern of oxygen consumption changes is consistent with phenomena of spontaneous permeabilization. Notably, in Elovl2 KO mitochondria, the spontaneous permeabilization has developed much quicker than in WT mitochondria (Figure 6D).

Since the magnitude of the CATR effect may reflect the amount of ANT in mitochondria, we examined ANT protein levels in isolated mitochondria analyzed as described above. Western blot analysis revealed that the amount of ANT2 (the liver-specific isoform) was higher in Elovl2 $\mathrm{KO}$ mitochondria than in WT (Figure 6EF). Western blot of ANT1 was also performed, but no clear band was detected (not shown).

Thus, fatty acid-induced uncoupled respiration in liver mitochondria is primarily mediated by ANT2. Furthermore, it seems that the contribution of this translocase in mitochondrial inefficiency is more significant in Elovl2 $\mathrm{KO}$ than in wild-type mitochondria. Also, interestingly, the higher amount of ANT2 coincides with higher content of VDAC (Figure $4 \mathrm{AB}$ ), indicating the potential cooperation of these transport proteins.

\section{Discussion}

\section{Possible mechanisms of impaired mitochondrial oxidative phosphorylation in PUFA deficient mitochondria}

The importance of lipid composition in the mitochondrial membrane for the electron transport chain and ATP synthesis has been suggested in various models [1-3,35]. Studied models in the liver are often pathologies (like hepatic steatosis) and linked to oxidative stress (high production of ROS) [35,36]. PUFAs are considered to play a central role in these pathologies (as well as aging and age-related diseases) since within the mitochondrial membrane, PUFAs are the primary cellular target of ROS attack and contribute to the generation of oxidatively damaged proteins $[16,17,37,38]$.

However, the Elovl2 $\mathrm{KO}$ mouse model studied here is an outstanding model. In contrast to the pathological mechanism described above of impairment of mitochondrial function via oxidatively modified mitochondrial PUFAs, the Elovl2 KO mitochondrial proteins are not damaged. Rather, the opposite was observed with the substantial decrease in the lipoxidation marker MDAL in the PUFA-deficient Elovl2 KO mice. Moreover, Elovl2 $\mathrm{KO}$ mice are resistant to hepatic steatosis induced by a high-fat diet [11]. Future studies of mitochondria in Elovl2 $\mathrm{KO}$ mice on a high-fat diet would help establish the possible link between resistance to oxidative damage and resistance to hepatic steatosis.

Regarding PUFA's role in aging, it is worth mentioning here the discovery of ELOVL2 gene as the most extreme example of age-related DNA hypermethylation in human whole blood DNA [39]. Further studies are needed to understand whether ELOVL2 hypermethylation only represents an indicator of chronological age or rather is functionally correlated with physiological status and specific clinical conditions.

Our study indicates that the deficiency in long-chain PUFAs (especially DHA), without modification of respiratory proteins, was sufficient to significantly reduce coefficients of respiratory control ratio (RCR), reflecting less coupled and less efficient oxidative phosphorylation in the Elovl2 KO mitochondria. This observation agrees with findings by $[5,18]$ of lower oxidative phosphorylation in phospholipid remodeling mitochondria. Although no changes were seen in respiratory protein quantity, fatty acid remodeling of mitochondrial membranes can affect the formation of respiratory chain 
supercomplexes [40,41] and thus reduce oxidative phosphorylation efficiency. Another explanation for this inefficiency could be conformational changes that affect the catalytic centers of mitochondrial complexes and decrease their activity [19,42].

\section{Mechanisms of mitochondrial inefficiency in genetically acquired PUFA-deficiency mice}

Our findings suggest that mitochondria could play an essential role in the metabolic changes observed in the Elovl2 KO mouse model [11,28]. The high mitochondrial volume revealed by electron microscopy and, consequently, high total basal mitochondrial proton leak could contribute to high basal metabolism [43,44].

Furthermore, high oligomycin-insensitive respiration reflecting basal proton leak at the mitochondrial level (independently of mitochondrial number) is another possible contributor to metabolic inefficiency of Elovl2 $\mathrm{KO}$ mice. There are ongoing debates in the literature about the mechanism of basal proton leak in mitochondria [43-47]. Three alternatives are suggested: the first, that proton leak is a pure mitochondrial membrane fatty acids/phospholipid characteristic; the second, that proton leak is a pure mitochondrial membrane protein (like UCP1) characteristic; and finally, that proton leak occurs via membrane proteins which are modified by surrounding phospholipids $[46,47]$. Aside from UCP1, adenine nucleotide translocase (ANT) is one of the membrane proteins that is involved in the mechanism of both basal and inducible mitochondrial proton leak [31,48-50]. Our earlier study [32] demonstrated that ANT1 is involved in basal proton leak (in agreement with [51]), but that ANT2 is involved in fatty acid-induced uncoupling. ANT1 is not present in the liver; thus, the high basal proton leak (oligomycin-insensitive oxygen consumption) in liver mitochondria isolated from Elovl2 $\mathrm{KO}$ may be explained by the first alternative mechanism, where proton leak is a pure mitochondrial membrane fatty acids/phospholipid characteristic. Thus, our results suggested that PUFAs-deficient mitochondria are leaky.

Concerning the fatty acid-inducible mitochondrial uncoupling, several mechanisms of action have been discussed, including ANT involvement [52]. The content of ANT2 is high in isolated liver mitochondria of Elovl2 $\mathrm{KO}$ mice. Moreover, mitochondrial volume is also high, meaning the total enhanced level of ANT2 could significantly contribute to Elovl2 $\mathrm{KO}$ mouse liver metabolism. However, it needs to be considered that ANT-mediated uncoupling can only be observed under in vivo conditions where the level of endogenous non-esterified fatty acids is sufficiently high (reaching the level added in-vitro). One such condition with high levels of non-esterified fatty acids due to hydrolysis of mitochondrial phospholipids was described for liver infection with F.hepatica [3]. Further research to identify additional pathologic or physiological in vivo conditions with elevated levels of non-esterified fatty acids is required to confirm the significance of ANT involvement in the mechanism of metabolic inefficiency.

\section{Indications of adaptive structural changes in liver mitochondria in genetically ac- quired PUFA-deficiency mice.}

In Elovl2 KO liver mitochondria, an upregulated level of the outer membrane protein VDAC was observed. VDAC is involved in numerous mitochondrial transport systems [22,53]. Our previous studies have shown outer membrane transport upregulation in mitochondria with respiratory chain deficiency [54]. In Elovl2 KO mice, the transport systems may respond to increased demand for lipid trafficking. Lipid exchange is essential for maintaining mitochondrial membranes, and it is driven at the endoplasmic reticulum-mitochondria juxtaposition [22,41,55]. Furthermore, endoplasmic reticulum-mitochondria contact sides change depending on food intake, i.e., increased under starvation but decreased in over-nutrition [21]. Such modification of endoplasmic reticulum-mitochondria contact sides could also happen in our model of PUFAs deficient mitochondria, which would be interesting to analyze to a deeper extent.

VDAC and ANT often cooperate as part of one transport complex through the inner and outer mitochondrial membrane [53]. Mitochondria with high levels of VDAC- 
ANT complexes are more prone to opening permeability transition pores and to apoptosis [56]. Observed patterns of spontaneous stimulation and following quick inhibition of oxygen consumption in Elovl2 KO liver mitochondria exposed to oleate and CATR may indicate easier ongoing permeabilization of the mitochondrial membranes. Such observation is consistent with [57] on CATR potentiation of spontaneous permeabilization in mitochondria with low membrane potential. The study of the detailed mechanism of such a phenomenon could be performed in future with more appropriate techniques.

\section{The physiological value of long chain PUFAs as nutrients}

Using a genetically acquired PUFA-deficiency mouse model, we showed the significance of polyunsaturated fatty acids (especially long-chain, DHA) for proper mitochondrial function. Furthermore, our results helped to unravel the mechanism for dietary effects identified in numerous previous studies $[1,2,5,58]$. The omega-6 PUFAs, 18:2n-6 and 20:4n-6, and the omega-3 PUFA, 22:6n-3 (DHA), are the most abundant long-chain PUFAs in the phospholipids pool of the mitochondrial membrane (DHA content is more than $6 \mathrm{~mol} \%$ of total mitochondrial lipids (Figure 2A)). Typical diets, however, contain relatively high amounts of 18:2n-6 and 20:4n-6 but are low in DHA content - especially the rodent chow diet, which lacks DHA typically. A study of Elovl2 KO mice has elucidated that the DHA within the liver mitochondrial membrane in chow-fed mice is endogenously derived via Elovl2 in the endoplasmic reticulum and peroxisomal fatty acid oxidation.

Thus, our study of a mouse model with genetically acquired fatty acid composition remodeling along with previous diet studies indisputably highlights the physiological value of PUFAs as nutrients. The knowledge presented here implies that endogenous long-chain PUFA production is vital for mice and human metabolism. Our findings are underlying several other studies on identifying genetic variants in the ELOVL2 gene associated with obesity-related conditions [7-9]. Dietetics and scholars need to consider interindividual genetic variability in endogenous PUFA modification in humans coupled with PUFA derived from the diet when the relationship between PUFA intake and effects is investigated. These findings support the genetics accounting for interindividual variability in molecular metabolism.

Supplementary Materials: The following are available online at www.mdpi.com/xxx/s1, Figure S1: Acyl chain length (ACL) of mitochondrial fatty acids from wild-type and Elovl2 KO mice; Figure S2: Relative level of oxidative stress biomarkers.

Author Contributions: A.G.R and I.G.S conceived and designed the study; E.T., A.G.R, I.G.S, R.P, A.M.P, A.V.K., G.B. and A.N. performed experiments and analyzed data; A.J. created the Elovl2 KO mice, R.P. and T.B. provided reagents and tools; A.G.R, E.T. and I.G.S wrote the paper. All authors discussed the results and commented on the manuscript.

Funding: This study was supported by grants from the Swedish Science Council (VR-NT 2017-04715 and VR-MT 2019-01508). This research was also funded in part by the Spanish Ministry of Science, Innovation, and Universities (grant RTI2018-099200-B-I00), and the Generalitat of Catalonia: Agency for Management of University and Research Grants (2017SGR696) to R.P. This study was co-financed by FEDER funds from the European Union ("A way to build Europe"). IRB Lleida is a CERCA Programme/Generalitat of Catalonia.' Open access funding was provided by Stockholm University, Sweden.

Institutional Review Board Statement: This study was approved by the Institutional Review Board on Animal Studies of Stockholm University and by the Animal Ethics Board of the North Stockholm region (protocol code 302/11 and date of 2014.08.25), and performed in accordance with national guidelines and regulations for the care and use of laboratory animals in agreement with the guidelines of the European Communities Council Directive 2010/63/EU for the care and use of laboratory animals. All efforts were made to minimize the number of animals used and their suffering. 
Acknowledgments: We thank Barbara Cannon, Jan Nedergaard and Valerio Chiurchiu' for valuable discussion and Christine Kallenberg for the English language editing. We thank the Experimental Core Facility (ECF), Stockholm University and the Electron Microscopy Unit (EMil) at Huddinge University hospital for technical assistance with the animals and TEM.

Conflicts of Interest: None of the authors have any competing interests in the manuscript.

Abbreviations: DHA, docosahexaenoic acid; ELOV2, elongation of very long-chain fatty acids 2; PUFA, polyunsaturated fatty acid; ROS, reactive oxygen species; VDAC, voltage-dependent anion channel; ANT, adenine nucleotide translocase; DPAn6, docosapentaenoic acid; MDAL, malondialdehyde-lysine; CEL, carboxyethyl lysine,; CML, carboxymethyl lysine; GSA, glutamic semialdehyde; AASA, aminoadipic semialdehyde; SFA, saturated fatty acids; UFA, unsaturated fatty acids; MUFA, monounsaturated fatty acids; PUFA polyunsaturated fatty acids; DBI, Double bond index; RCR, Respiratory Control Ratio; CATR, carboxyatractyloside; FCCP, carbonyl cyanide p-trifluoromethoxy phenylhydrazone; OXPHOS, oxidative phosphorylation.

\section{References}

1. Stanley, W.C.; Khairallah, R.J.; Dabkowski, E.R. Update on lipids and mitochondrial function: Impact of dietary n-3 polyunsaturated fatty acids. Current opinion in clinical nutrition and metabolic care 2012, 15, 122-126.

2. Robblee, N.M.; Clandinin, M.T. Effect of dietary fat level and polyunsaturated fatty acid content on the phospholipid composition of rat cardiac mitochondrial membranes and mitochondrial atpase activity. J Nutr 1984, 114, 263-269.

3. Lenton, L.M.; Behm, C.A.; Bygrave, F.L. Aberrant mitochondrial respiration in the livers of rats infected with fasciola hepatica: The role of elevated non-esterified fatty acids and altered phospholipid composition. Biochem J 1995, 307 ( Pt 2), 425-431.

4. Yamaoka, S.; Urade, R.; Kito, M. Cardiolipin molecular species in rat heart mitochondria are sensitive to essential fatty acid-deficient dietary lipids. J Nutr 1990, 120, 415-421.

5. Yamaoka, S.; Urade, R.; Kito, M. Mitochondrial function in rats is affected by modification of membrane phospholipids with dietary sardine oil. J. Nutr. 1988, 118, 290-296.

6. Guillou, H.; Zadravec, D.; Martin, P.G.; Jacobsson, A. The key roles of elongases and desaturases in mammalian fatty acid metabolism: Insights from transgenic mice. Prog Lipid Res 2010, 49, 186-199.

7. Maguolo, A.; Zusi, C.; Giontella, A.; Miraglia Del Giudice, E.; Tagetti, A.; Fava, C.; Morandi, A.; Maffeis, C. Influence of genetic variants in fads2 and elovl2 genes on bmi and pufas homeostasis in children and adolescents with obesity. International Journal of Obesity 2021, 45, 56-65.

8. Alsaleh, A.; Maniou, Z.; Lewis, F.J.; Hall, W.L.; Sanders, T.A.; O'Dell, S.D. Elovl2 gene polymorphisms are associated with increases in plasma eicosapentaenoic and docosahexaenoic acid proportions after fish oil supplement. Genes Nutr 2014, 9, 362.

9. de la Garza Puentes, A.; Montes Goyanes, R.; Chisaguano Tonato, A.M.; Torres-Espínola, F.J.; Arias García, M.; de Almeida, L.; Bonilla Aguirre, M.; Guerendiain, M.; Castellote Bargalló, A.I.; Segura Moreno, M., et al. Association of maternal weight with fads and elovl genetic variants and fatty acid levels- the preobe follow-up. PLoS One 2017, 12, e0179135.

10. Zadravec, D.; Tvrdik, P.; Guillou, H.; Haslam, R.; Kobayashi, T.; Napier, J.A.; Capecchi, M.R.; Jacobsson, A. Elovl2 controls the level of $n-628: 5$ and 30:5 fatty acids in testis, a prerequisite for male fertility and sperm maturation in mice. Journal of lipid research 2011, 52, 245-255.

11. Pauter, A.M.; Olsson, P.; Asadi, A.; Herslof, B.; Csikasz, R.I.; Zadravec, D.; Jacobsson, A. Elovl2 ablation demonstrates that systemic dha is endogenously produced and is essential for lipid homeostasis in mice. Journal of lipid research 2014, 55, 718-728.

12. Cullis, P.R.; de Kruijff, B. Lipid polymorphism and the functional roles of lipids in biological membranes. Biochim Biophys Acta 1979, 559, 399-420.

13. Gohil, V.M.; Greenberg, M.L. Mitochondrial membrane biogenesis: Phospholipids and proteins go hand in hand. The Journal of cell biology 2009, 184, 469-472.

14. Mejia, E.M.; Hatch, G.M. Mitochondrial phospholipids: Role in mitochondrial function. J Bioenerg Biomembr 2016, 48, 99-112.

15. Pratt, D.A.; Tallman, K.A.; Porter, N.A. Free radical oxidation of polyunsaturated lipids: New mechanistic insights and the development of peroxyl radical clocks. Acc Chem Res 2011, 44, 458-467.

16. Al-Gubory, K.H. Mitochondria: Omega-3 in the route of mitochondrial reactive oxygen species. Int J Biochem Cell Biol 2012, 44, 1569-1573. 
17. Pamplona, R.; Dalfo, E.; Ayala, V.; Bellmunt, M.J.; Prat, J.; Ferrer, I.; Portero-Otin, M. Proteins in human brain cortex are modified by oxidation, glycoxidation, and lipoxidation. Effects of alzheimer disease and identification of lipoxidation targets. J Biol Chem 2005, 280, 21522-21530.

18. Tasseva, G.; Bai, H.D.; Davidescu, M.; Haromy, A.; Michelakis, E.; Vance, J.E. Phosphatidylethanolamine deficiency in mammalian mitochondria impairs oxidative phosphorylation and alters mitochondrial morphology. J Biol Chem 2013, 288, 4158-4173.

19. Hoch, F.L. Cardiolipins and mitochondrial proton-selective leakage. J Bioenerg Biomembr 1998, 30, 511-532.

20. Osman, C.; Voelker, D.R.; Langer, T. Making heads or tails of phospholipids in mitochondria. The Journal of cell biology 2011, 192, 7-16.

21. Horvath, S.E.; Daum, G. Lipids of mitochondria. Prog Lipid Res 2013, 52, 590-614.

22. Flis, V.V.; Daum, G. Lipid transport between the endoplasmic reticulum and mitochondria. Cold Spring Harb Perspect Bio/ 2013, 5.

23. Shabalina, I.G.; Jacobsson, A.; Cannon, B.; Nedergaard, J. Native ucp1 displays simple competitive kinetics between the regulators purine nucleotides and fatty acids. J Biol Chem 2004, 279, 38236-38248.

24. Udenfriend, S.; Stein, S.; Bohlen, P.; Dairman, W.; Leimgruber, W.; Weigele, M. Fluorescamine: A reagent for assay of amino acids, peptides, proteins, and primary amines in the picomole range. Science 1972, 178, 871-872.

25. Caro, P.; Gomez, J.; Sanchez, I.; Naudi, A.; Ayala, V.; Lopez-Torres, M.; Pamplona, R.; Barja, G. Forty percent methionine restriction decreases mitochondrial oxygen radical production and leak at complex i during forward electron flow and lowers oxidative damage to proteins and mitochondrial DNA in rat kidney and brain mitochondria. Rejuvenation Res 2009, 12, 421-434.

26. Bokhari, M.H.; Halleskog, C.; Aslund, A.; Boulet, N.; Casadesus Rendos, E.; de Jong, J.M.A.; Csikasz, R.; Amri, E.Z.; Shabalina, I.; Bengtsson, T. Isothermal microcalorimetry measures ucp1-mediated thermogenesis in mature brite adipocytes. Commun Bio/2021, 4, 1108.

27. Weibel, E.R.; Paumgartner, D. Integrated stereological and biochemical studies on hepatocytic membranes. li. Correction of section thickness effect on volume and surface density estimates. The Journal of cell biology 1978, 77, 584-597.

28. Pauter, A.M.; Fischer, A.W.; Bengtsson, T.; Asadi, A.; Talamonti, E.; Jacobsson, A. Synergistic effects of dha and sucrose on body weight gain in pufa-deficient elovl2 -/- mice. Nutrients 2019, 11.

29. Hou, L.; Lian, K.; Yao, M.; Shi, Y.; Lu, X.; Fang, L.; He, T.; Jiang, L. Reduction of n-3 pufas, specifically dha and epa, and enhancement of peroxisomal beta-oxidation in type 2 diabetic rat heart. Cardiovasc Diabeto/2012, 11, 126.

30. Panov, A.; Filippova, S.; Lyakhovich, V. Adenine nucleotide translocase as a site of regulation by adp of the rat liver mitochondria permeability to h+ and k+ ions. Arch Biochem Biophys 1980, 199, 420-426.

31. Andreyev, A.; Bondareva, T.O.; Dedukhova, V.I.; Mokhova, E.N.; Skulachev, V.P.; Tsofina, L.M.; Volkov, N.I.; Vygodina, T.V. The atp/adp-antiporter is involved in the uncoupling effect of fatty acids on mitochondria. Eur J Biochem 1989, 182, 585-592.

32. Shabalina, I.G.; Kramarova, T.V.; Nedergaard, J.; Cannon, B. Carboxyatractyloside effects on brown-fat mitochondria imply that the adenine nucleotide translocator isoforms ant 1 and ant2 may be responsible for basal and fatty-acid-induced uncoupling respectively. Biochem J 2006, 399, 405-414

33. Winkler, H.H.; Bygrave, F.L.; Lehninger, A.L. Characterization of the atractyloside-sensitive adenine nucleotide transport system in rat liver mitochondria. J Biol Chem 1968, 243, 20-28.

34. Pebay-Peyroula, E.; Dahout-Gonzalez, C.; Kahn, R.; Trezeguet, V.; Lauquin, G.J.; Brandolin, G. Structure of mitochondrial adp/atp carrier in complex with carboxyatractyloside. Nature 2003, 426, 39-44.

35. Garnol, T.; Endlicher, R.; Kučera, O.; Drahota, Z.; Červinková, Z. Impairment of mitochondrial function of rat hepatocytes by high fat diet and oxidative stress. Physiological Research 2014, 63, 271-274.

36. Simões, I.C.M.; Fontes, A.; Pinton, P.; Zischka, H.; Wieckowski, M.R. Mitochondria in non-alcoholic fatty liver disease. Int J Biochem Cell Bio/2018, 95, 93-99.

37. Pamplona, R.; Barja, G. An evolutionary comparative scan for longevity-related oxidative stress resistance mechanisms in homeotherms. Biogerontology 2011, 12, 409-435. 
38. Giorgi, C.; Marchi, S.; Simoes, I.C.M.; Ren, Z.; Morciano, G.; Perrone, M.; Patalas-Krawczyk, P.; Borchard, S.; Jędrak, P.; Pierzynowska, K., et al. Mitochondria and reactive oxygen species in aging and age-related diseases. Int Rev Cell Mol Biol 2018, 340, 209-344.

39. Garagnani, P.; Bacalini, M.G.; Pirazzini, C.; Gori, D.; Giuliani, C.; Mari, D.; Di Blasio, A.M.; Gentilini, D.; Vitale, G.; Collino, S., et al. Methylation of elovl2 gene as a new epigenetic marker of age. Aging Cel/ 2012, 11, 1132-1134.

40. Milenkovic, D.; Blaza, J.N.; Larsson, N.G.; Hirst, J. The enigma of the respiratory chain supercomplex. Cell Metab 2017, 25, 765-776.

41. Szymański, J.; Janikiewicz, J.; Michalska, B.; Patalas-Krawczyk, P.; Perrone, M.; Ziółkowski, W.; Duszyński, J.; Pinton, P.; Dobrzyń, A.; Więckowski, M.R. Interaction of mitochondria with the endoplasmic reticulum and plasma membrane in calcium homeostasis, lipid trafficking and mitochondrial structure. Int J Mol Sci 2017, 18.

42. Lenaz, G.; Genova, M.L. Supramolecular organisation of the mitochondrial respiratory chain: A new challenge for the mechanism and control of oxidative phosphorylation. Advances in experimental medicine and biology 2012, 748, 107-144.

43. Brand, M.D.; Chien, L.F.; Ainscow, E.K.; Rolfe, D.F.; Porter, R.K. The causes and functions of mitochondrial proton leak. Biochim. Biophys. Acta. 1994, 1187, 132-139.

44. Porter, R.K. Allometry of mammalian cellular oxygen consumption. Cell Mol Life Sci 2001, 58, 815-822.

45. Shabalina, I.G.; Nedergaard, J. Mitochondrial ('mild') uncoupling and ros production: Physiologically relevant or not? Biochem Soc Trans 2011, 39, 1305-1309.

46. Divakaruni, A.S.; Brand, M.D. The regulation and physiology of mitochondrial proton leak. Physiology (Bethesda, Md.) 2011, 26, 192-205.

47. Nicholls, D.G. Mitochondrial proton leaks and uncoupling proteins. Biochim Biophys Acta Bioenerg 2021, 1862, 148428.

48. Brustovetsky, N.N.; Dedukhova, V.I.; Egorova, M.V.; Mokhova, E.N.; Skulachev, V.P. Inhibitors of the atp/adp antiporter suppress stimulation of mitochondrial respiration and $\mathrm{h}+$ permeability by palmitate and anionic detergents. FEBS Lett 1990, 272, 187-189.

49. Bertholet, A.M.; Chouchani, E.T.; Kazak, L.; Angelin, A.; Fedorenko, A.; Long, J.Z.; Vidoni, S.; Garrity, R.; Cho, J.; Terada, N., et al. $\mathrm{H}(+)$ transport is an integral function of the mitochondrial adp/atp carrier. Nature 2019, 571, 515-520.

50. Kreiter, J.; Rupprecht, A.; Skulj, S.; Brkljaca, Z.; Zuna, K.; Knyazev, D.G.; Bardakji, S.; Vazdar, M.; Pohl, E.E. Ant1 activation and inhibition patterns support the fatty acid cycling mechanism for proton transport. Int J Mol Sci2021, 22.

51. Brand, M.D.; Pakay, J.L.; Ocloo, A.; Kokoszka, J.; Wallace, D.C.; Brookes, P.S.; Cornwall, E.J. The basal proton conductance of mitochondria depends on adenine nucleotide translocase content. Biochem J 2005, 392, 353-362.

52. Nedergaard, J.; Golozoubova, V.; Matthias, A.; Asadi, A.; Jacobsson, A.; Cannon, B. Ucp1: The only protein able to mediate adaptive non-shivering thermogenesis and metabolic inefficiency. Biochim. Biophys. Acta 2001, 1504, 82-106.

53. Vyssokikh, M.Y.; Brdiczka, D. The function of complexes between the outer mitochondrial membrane pore (vdac) and the adenine nucleotide translocase in regulation of energy metabolism and apoptosis. Acta Biochim Po/ 2003, 50, 389-404.

54. Shabalina, I.G.; Landreh, L.; Edgar, D.; Hou, M.; Gibanova, N.; Atanassova, N.; Petrovic, N.; Hultenby, K.; Soder, O.; Nedergaard, J., et al. Leydig cell steroidogenesis unexpectedly escapes mitochondrial dysfunction in prematurely aging mice. FASEB J 2015, 29, 3274-3286.

55. Tatsuta, T.; Scharwey, M.; Langer, T. Mitochondrial lipid trafficking. Trends Cell Bio/ 2014, 24, 4452.

56. Zhivotovsky, B.; Galluzzi, L.; Kepp, O.; Kroemer, G. Adenine nucleotide translocase: A component of the phylogenetically conserved cell death machinery. Cel/ Death Differ 2009, 16, 1419-1425.

57. Halestrap, A.P.; Woodfield, K.Y.; Connern, C.P. Oxidative stress, thiol reagents, and membrane potential modulate the mitochondrial permeability transition by affecting nucleotide binding to the adenine nucleotide translocase. J Bio/ Chem 1997, 272, 3346-3354.

58. Červinková, Z.; Drahota, Z. Enteral administration of lipid emulsions protects liver cytochrome $c$ oxidase from hepatotoxic action of thioacetamide. Physiological Research 1998, 47, 151-154. 